\title{
Transformer-CNN: Swiss knife for QSAR modeling and interpretation
}

\author{
Pavel Karpov ${ }^{1,2^{*}}$, Guillaume Godin ${ }^{3}$ and Igor V. Tetko ${ }^{1,2}$
}

\begin{abstract}
We present SMILES-embeddings derived from the internal encoder state of a Transformer [1] model trained to canonize SMILES as a Seq2Seq problem. Using a CharNN [2] architecture upon the embeddings results in higher quality interpretable QSAR/QSPR models on diverse benchmark datasets including regression and classification tasks. The proposed Transformer-CNN method uses SMILES augmentation for training and inference, and thus the prognosis is based on an internal consensus. That both the augmentation and transfer learning are based on embeddings allows the method to provide good results for small datasets. We discuss the reasons for such effectiveness and draft future directions for the development of the method. The source code and the embeddings needed to train a QSAR model are available on https://github.com/bigchem/transformer-cnn. The repository also has a standalone program for QSAR prognosis which calculates individual atoms contributions, thus interpreting the model's result. OCHEM [3] environment (https://ochem .eu) hosts the on-line implementation of the method proposed.
\end{abstract}

Keywords: Transformer model, Convolutional neural neural networks, Augmentation, QSAR, SMILES, Embeddings, Character-based models, Cheminformatics, Regression, Classification

\section{Introduction}

Quantitative Structure-Activity (Property) Relationship (QSAR/QSPR) approaches find a nonlinear function, often modelled as an artificial neural network (ANN), that estimates the activity/property based on a chemical structure. In the past, most QSAR works heavily relied on descriptors [4] that represent in a numerical way some features of a complex graph structure of a compound. Amongst numerous families of descriptors, the fragment descriptors that count occurrences of a subgraph in a molecule graph, hold a distinctive status due to simplicity in the calculation. Moreover, there is a theoretical proof that one can successfully build any QSAR model with them [5]. Even a small database of compounds contains thousands of fragmental descriptors and some feature selection algorithm

\footnotetext{
*Correspondence: pavel.karpov@helmholtz-muenchen.de ${ }^{1}$ Institute of Structural Biology, Helmholtz Zentrum München-Research Center for Environmental Health $(\mathrm{GmbH})$, Ingolstädter Landstraße 1, 85764 Neuherberg, Germany

Full list of author information is available at the end of the article
}

has traditionally been used to find a proper subset of descriptors for better quality, and to speed up the whole modeling process. Thus, feature selection in conjunction with a suitable machine learning method was key to success [6]. The rise of deep learning [7] allows us to bypass tiresome expert and domain-wise feature construction by delegating this task to a neural network that can extract the most valuable traits of the raw input data required for modeling the problem at hand $[8,9]$.

In this setting, the whole molecule as a SMILES-strings $[10,11]$ (Simplified Molecular Input Line Entry System) or a graph $[12,13]$ serves as the input to the neural network. SMILES notation allows for the writing of any complex formula of an organic compound in a string facilitating storage and retrieval information about molecules in databases [14]. It contains all information about the compound sufficient to derive the entire configuration (3D-structure) and has a direct connection to the nature of fragmental descriptors, Fig. 1, thus, making SMILES one of the best representation for QSAR studies.

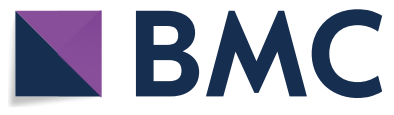

(c) The Author(s) 2020. This article is licensed under a Creative Commons Attribution 4.0 International License, which permits use, sharing, adaptation, distribution and reproduction in any medium or format, as long as you give appropriate credit to the original author(s) and the source, provide a link to the Creative Commons licence, and indicate if changes were made. The images or other third party material in this article are included in the article's Creative Commons licence, unless indicated otherwise in a credit line to the material. If material is not included in the article's Creative Commons licence and your intended use is not permitted by statutory regulation or exceeds the permitted use, you will need to obtain permission directly from the copyright holder. To view a copy of this licence, visit http://creativeco mmons.org/licenses/by/4.0/. The Creative Commons Public Domain Dedication waiver (http://creativecommons.org/publicdomain/ zero/1.0/) applies to the data made available in this article, unless otherwise stated in a credit line to the data. 


\section{Canonical $\quad[[\mathrm{C} @ H] 12 \mathrm{SC}(\mathrm{C})(\mathrm{C})[\mathrm{C} @ @ H](\mathrm{N} 1 \mathrm{C}(=\mathrm{O})[\mathrm{C} @ \mathrm{H}] 2 \mathrm{NC}(=\mathrm{O}) \mathrm{Cc} 1 \mathrm{ccccc} 1) \mathrm{C}(=\mathrm{O}) \mathrm{O}$}
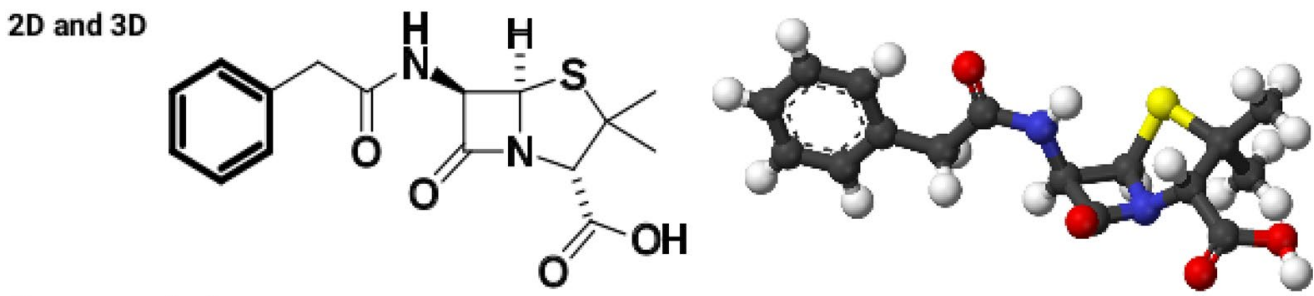

\section{Non-canonical}

$\mathrm{C} 1(=0)[\mathrm{C} @ H]([\mathrm{C} @ H] 2 \mathrm{SC}(\mathrm{C})(\mathrm{C})[\mathrm{C} @ @ H](\mathrm{N} 12) \mathrm{C}(=0) 0) \mathrm{NC}(=0) \mathrm{Cc} 1 \mathrm{ccccc} 1$ $\mathrm{O}=\mathrm{C}([\mathrm{C} @ \mathrm{H}] 1 \mathrm{C}(\mathrm{S}[\mathrm{C} @ @ \mathrm{H}] 2[\mathrm{C} @ @ H](\mathrm{C}(=\mathrm{O}) \mathrm{N} 12) \mathrm{NC}(=\mathrm{O}) \mathrm{Cc} 1 \operatorname{ccccc} 1)(\mathrm{C}) \mathrm{C}) \mathrm{O}$ $\mathrm{C} 1(=0)[\mathrm{C} @ @ H](\mathrm{NC}(=\mathrm{O}) \mathrm{Cc} 2 \mathrm{ccccc} 2)[\mathrm{C} @ @ H] 2 \mathrm{~N} 1[\mathrm{C} @ \mathrm{H}](\mathrm{C}(\mathrm{C})(\mathrm{C}) \mathrm{S} 2) \mathrm{C}(=\mathrm{O}) \mathrm{O}$ etc.

Fig. 1 Benzylpenicillin canonical SMILES at the top, 2D and 3D structures derived from SMILES with OpenBabel [15] in the middle, and three non-canonical SMILES examples at the bottom. A substructure of the phenyl ring is written in bold font

One of the first works exploiting direct SMILES input as descriptors used fragmentation of strings into groups of overlapping substrings forming a SMILES-like set or a hologram of a molecule [16]. Within this approach, there was no need to derive a $2 \mathrm{D} / 3 \mathrm{D}$ configuration of the molecule with subsequent calculation of descriptors keeping the quality of the models at the same level as with classical descriptors or even better.

SMILES strings are sequences of characters; therefore, they can be analyzed by machine-learning methods suitable for text processing, namely with convolutional and recurrent neural networks. After the demonstration of text understanding from character-level inputs [17], this technique was adopted in chemoinformatics [11, 18-21]. Recently, we showed that the augmentation of SMILES (using canonical as well as non-canonical SMILES during model training and inference) increases the performance of convolutional models for regression and classification tasks [22].

Technically modern machine-learning models consist of two parts working together. The first part encodes the input data and extracts the most robust features by applying convolutional filters with different receptive fields (RF) or recurrent layers, whereas the second part directly builds the regular model based on these features using standard dense layers as building blocks (so called classical "MLP"), Fig. 2. Though powerful convolutional layers can effectively encode the input within its internal representation, usually one needs a considerable training dataset and computational resources to train the encoder part of a network.

The concept of embeddings mitigates the problem by using the pre-trained weights designed for image [23] or text processing [24] tasks. It allows transfer learning from previous data and speeds up the training process for building models with significantly smaller datasets inaccessible for training from scratch. Typically, QSAR datasets contain only several hundreds of molecules, and SMILES-embeddings could improve models by developing better features.

One way of separately obtaining SMILES embeddings is to use classical autoencoder [25] approach where the input is the same as the output. In the case of SMILES, however, it would be more desirable to explore a variety of SMILES belonging to the same molecule due to redundant SMILES grammar, Fig. 1. We hypothesized that it is possible to train a neural network to conduct a SMILES canonicalization task in a Sequence-to-Sequence (Seq2Seq) manner like a machine translation problem, where on the left side are non-canonical SMILES, and on the right side are their canonical equivalents. Recently, Seq2Seq was successfully applied to translation from InChi [26] codes to SMILES (Inchi2Sml) as well as from SMILES arbitrary to canonical SMILES (Sml2canSml), and to build QSAR models on extracted latent variables [27].

The state-of-the-art neural architecture for machine translation consists of stacked Long Short-Term Memory (LSTM) cells [28]. The training process for such networks inherently has all kinds of Recurrent Neural Networks difficulties, e.g., vanishing gradients, and the impossibility of parallelization. Recently, a Transformer model [1] was proposed where all recurrent units are replaced with convolutional and element-wise feed-forward layers. The whole architecture shows a significant speed-up during training and inference with improved accuracy over 


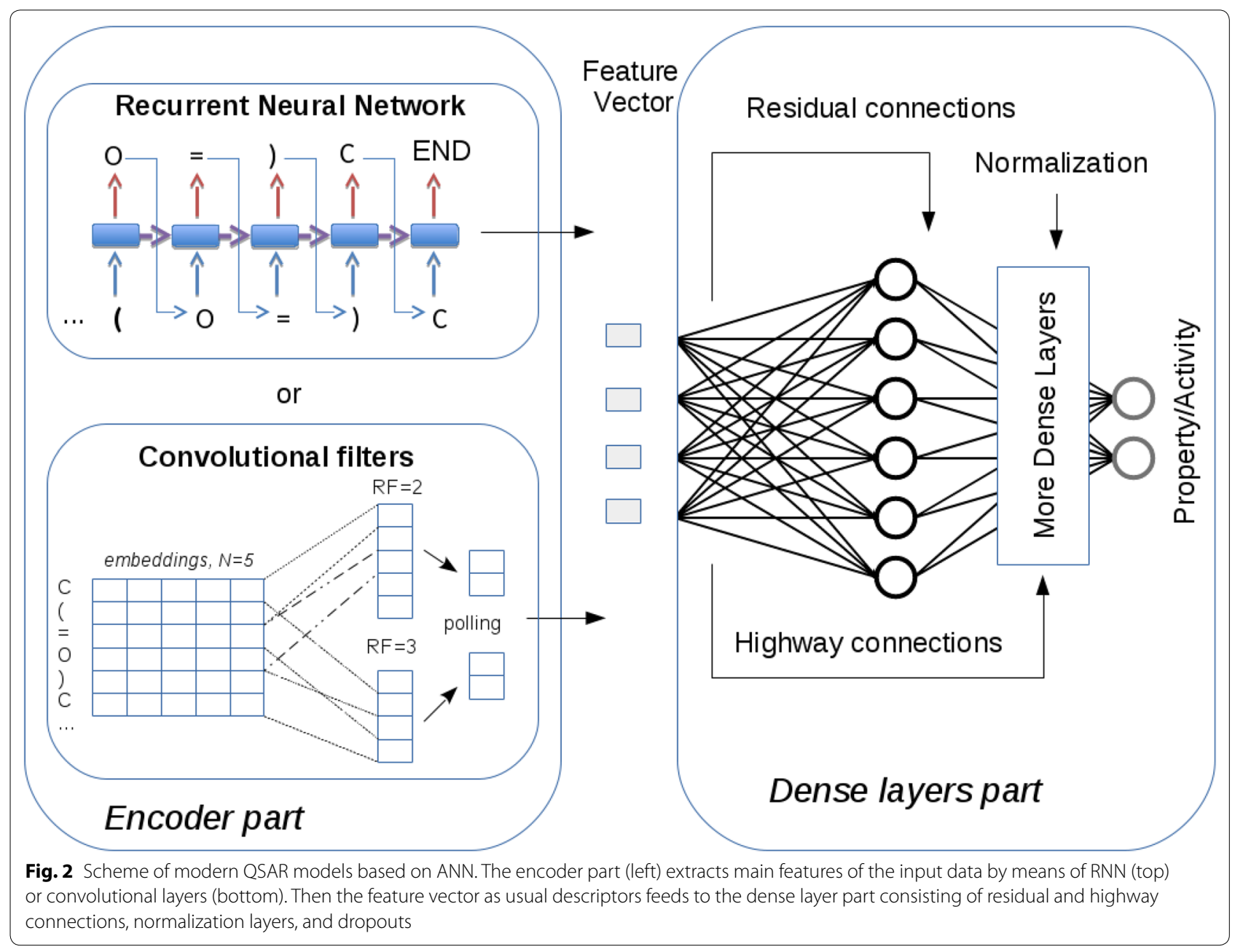

translation benchmarks. The Transformer model was applied for prediction of reaction outcomes [29] and for retrosynthesis [30].

Modern machine learning architectures although demonstrating incredible performance still lack interpretability. Explaining the reasons for a particular prediction of a model avoids "Clever Hans" predictors with spurious or non-relevant correlations [31] and foster trust and verifiability. One of the promising methods to open a "black box" uses the Layer-wise Relevance Propagation (LRP) algorithm [32], which splits the overall predicted value to a sum of contributions of individual neurons. In this method, the sum of relevance of all neurons of a layer, including the bias neuron, is kept constant. Propagation of the relevance from the last layer to the input layer allows the evaluation of the contributions of particular input features in to select the most relevant features for the whole training set [33] or to explain the individual neural network prediction [32]. We apply the LRP method for an explanation of individual results, checking the model get results for the right reason.

Our contributions in the article are as follows:

Presenting a concept of dynamic SMILES embeddings that may be useful for a wide range of cheminformatics tasks;

Scrutinizing CharNN models based on these embeddings for regression and classification tasks and show that the method outperforms the state-of-the-art models;

Interpretation of the model based on LRP method;

Our implementation as well as source codes and SMILES-embeddings are available on https://githu b.com/bigchem/transformer-cnn. We also provide ready-to-use implementation on https://ochem.eu within the OCHEM [3] environment and a standalone program for calculating properties and explaining the results. 


\section{Methods \\ SMILES canonicalization model Dataset}

To train the ANN to perform SMILES canonicalization, we used the ChEMBL database [34] with SMILES strings of length less than or equal 110 characters (>93\% of the entire database). The original dataset was augmented 10 times up to $17,657,995$ canonicalization pairs written in reactions format separated by ' $>>$ '. Each pair contained on the left side a non-canonical, and on the right side-a canonical SMILES for the same molecule. Such an arrangement of the training dataset allowed us to reuse the previous Transformer code, which was originally applied for retrosynthetic tasks [30]. For completeness, we added for every compound a line where both left and right sides were identical, i.e. canonical SMILES, Fig. 3. Thus each molecule was present in the training set 11 times. If a molecule had tautomeric forms then all of them were accounted for as separate entries in the training data file.

\section{Model input}

Seq2Seq models use one-hot encoding vector for the input. Its values are zero everywhere except the position of the current token which is set to one. Many works on SMILES use tokenization procedure $[35,36]$ that combines some characters, for example ' $\mathrm{B}$ ' and ' $\mathrm{r}$ ' to one token 'Br'. Other rules for handling most common two-letters elements, charges, and stereochemistry also are used for preparing the input for the neural network. According to our experience, the use of more complicated schemes instead of simple character-level tokenization did not increase the accuracy of models [30]. Therefore a simple character-level tokenization was used in this study. The vocabulary of our model consisted of all possible characters from ChEMBL dataset and has 66 symbols: $\wedge \# \%()+-. / 0123456789=@ A B C D E F G H I K L M N O P R S T-$ VXYZ[\]abcdefgilmnoprstuy\$
Thus, the model could handle the entire diversity of drug-like compounds including stereochemistry, different charges, and inorganic ions. Two special characters were added to the vocabulary: ' $\wedge$ ' to indicate the start of the sequence, and ' $\$$ ' to inform the model of the end of data input.

\section{Transformer model}

The canonicalization model used in this work was based upon a Transformer architecture consisting of two separate stacks of layers for the encoder and the decoder, respectively. Each layer incorporated some portion of knowledge written in its internal memory (V) with indexed access by keys (K). When new data arrived (Q), the layer calculated attention and modified the input accordingly (see the original work on Transformers [1]), thus, forming the output of the self-attention layer and weighting those parts that carry the essential information. Besides a self-attention mechanism, the layer also contained several position-wise dense layers, a normalization layer, and residual connections [1, 37]. Our model utilized a three layer architecture of Transformer with 10 blocks of self-attention, i.e. the same one as used in our previous study [30]. After the encoding process was finished, the output of the top encoder layer contained a representation of a molecule suitable for decoding into canonical SMILES. In this study we used this representation as a well-prepared latent representation for QSAR modeling.

Tensorflow v1.12.02 [38] was used as machine-learning framework to develop all parts of the Transformer, whereas RDKit v.2018.09.2 [39] was used for SMILES canonicalization and augmentation.

\section{QSAR model}

We call the output of the Transformer's encoder part a dynamic SMILES-embedding, Fig. 4. For a molecule with $N$-characters, the encoder produces the matrix with dimensions ( $N, E M B E D D I N G S)$. Though technically this

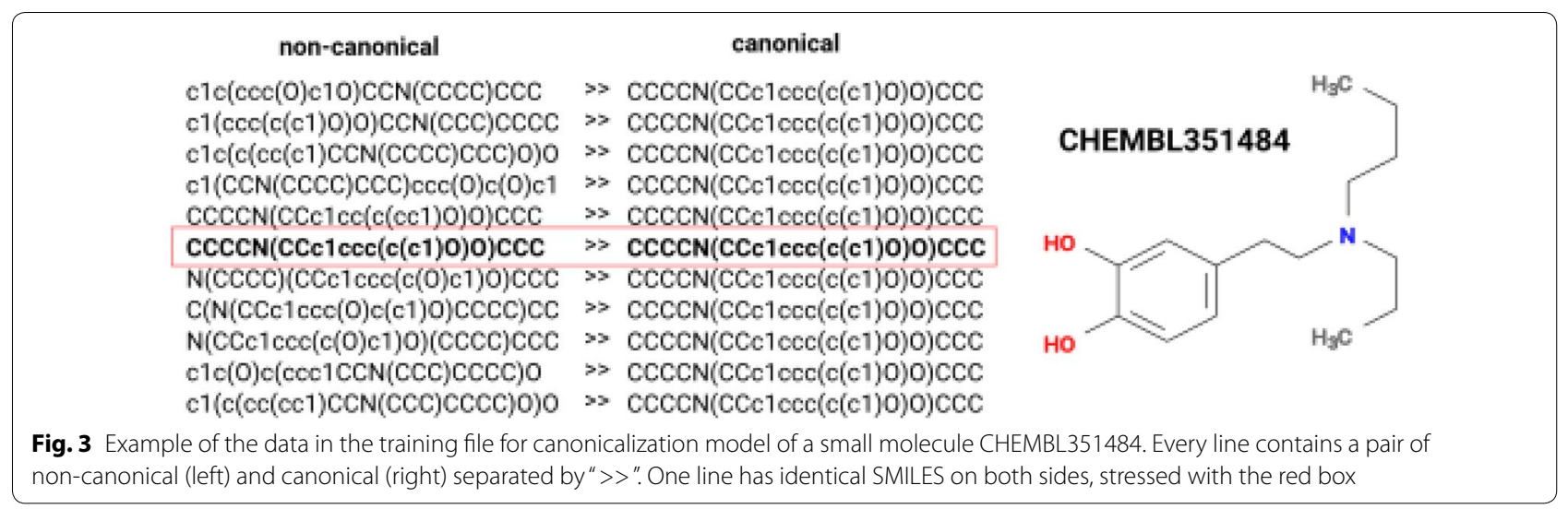




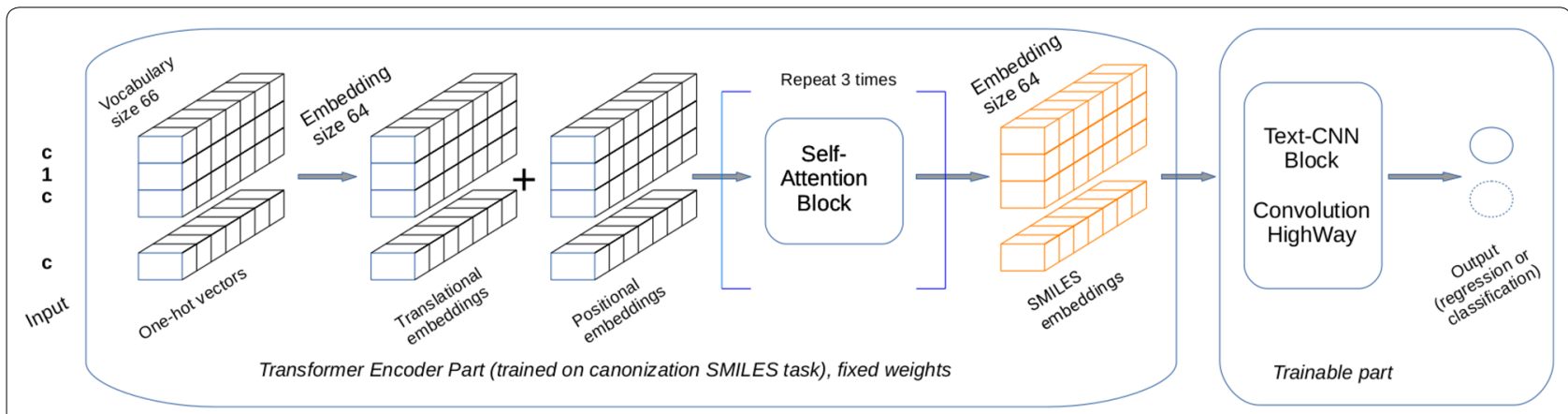

Fig. 4 The architecture of the Transformer-CNN network

matrix is not an embedding because equivalent characters have different values depending on position and surroundings, it can be considered so due to its role: to convert an input one-hot raw vectors to real-value vectors in some latent space. Because these embeddings have variable lengths, we used a series of 1D convolutional filters as implemented in DeepChem [40] TextCNN method (https://github.com/deepchem).

Each convolution had a kernel size from the list $(1,2,3$, $4,5,6,7,8,9,10,15,20)$ and produced the following number of filters (100, 200, 200, 200, 200, 100, 100, 100, 100, $100,160,160)$, respectively. After a GlobalMaxPool operation and the subsequent concatenation of the pooling results, the data went through Dropout [41] $($ rate $=0.25)$, Dense $(\mathrm{N}=512)$, Highway [42] layers, and, finally, converted to the output layer which consisted of only one neuron for regression and two neurons for classification tasks. The weights of the Transformer's part were frozen in all experiments. All models used the Adam optimizer with Mean Squared Error or Binary Cross-Entropy loss depending on the problem at hand. A fixed learning rate $\lambda=10^{-4}$ was used. Early-stopping was used to prevent overfitting, to select a best model, and to reduce training time. OCHEM calculations were performed using canonical SMILES as well as ten-fold augmented SMILES during both training and prognosis. This number of SMILES augmentations was found to be an optimal one in our previous study [43]. An average value of the individual predictions for different representation of the same molecule was used as the final model prediction to calculate statistical parameters.

The same five-fold cross-validation procedure was used to compare the models with the results of our previous study [43]. The coefficients of determination [44]

$$
r^{2}=1-S S_{\text {res }} / S S_{\text {tot }}
$$

where $\mathrm{SS}_{\text {tot }}$ is total variance of data and $\mathrm{SS}_{\text {res }}$ is residual unexplained variance of data was used to compare regression models and Area Under the Curve (AUC) was used for classification tasks.

\section{Validation datasets}

We used the same datasets ( 9 for regression and 9 for classification) that were exploited in our previous studies [11, 22]. Short information about these sets as well as links to original works are provided in Table 1 . The datasets are

Table 1 Descriptions of datasets used in the work

\begin{tabular}{|c|c|c|c|c|c|}
\hline Code & Description & Size & Code & Description & Size \\
\hline Regression tasks & & & Classification tasks & & \\
\hline MP & Melting point [45] & 19,104 & HIV & Inhibition of HIV replication [46] & 41,127 \\
\hline $\mathrm{BP}$ & Boiling point $[47]$ & 11,893 & AMES & Mutagenicity [48] & 6542 \\
\hline $\mathrm{BCF}$ & Bioconcentration factor [47] & 378 & BACE & Human $\beta$-secretase 1 (BACE-1) inhibitors [46] & 1513 \\
\hline FreeSolv & Free solvation energy [46] & 642 & Clintox & Clinical trial toxicity [46] & 1478 \\
\hline $\log S$ & Solubility [49] & 1311 & Tox21 & In-vitro toxicity [46] & 7831 \\
\hline Lipo & Lipophilicity [50] & 4200 & BBBP & Blood-brain barrier [46] & 2,039 \\
\hline BACE & $\begin{array}{l}\text { IC50 of human } \beta \text {-secretase } 1 \text { (BACE-1) } \\
\text { inhibitors }[46]\end{array}$ & 1513 & JAK3 & Janus kinase 3 inhibitor [51] & 886 \\
\hline DHFR & Dihydrofolate reductase inhibition [52] & 739 & BioDeg & Biodegradability [53] & 1737 \\
\hline LEL & Lowest effect level [54] & 483 & $\mathrm{RP} A \mathrm{R}$ & Endocrine disruptors [55] & 930 \\
\hline
\end{tabular}


available on the OCHEM environment on https://ochem .eu.

\section{Results and discussion SMILES canonicalization model}

The Transformer model was trained for 10 epochs with the learning rate changing according to the formula:

$$
\lambda=\text { factor } * \min (1.0, \text { step } / \text { warmup }) / \max (\text { step }, \text { warmup })
$$

where factor $=20$, warmup $=16,000$ steps, and if $\lambda<10^{-4}$ then $\lambda=10^{-4}$. The settings for the learning rate were similar to those used in our retro-synthesis study. Each epoch contained 275,907 steps (batches). No early-stopping or weight-averaging was applied. Learning curves are shown in Fig. 5.

To validate the model, we sampled 500,000 ChEMBLlike SMILES (only 8,617 (1.7\%) of them were canonical) from a generator [56] and checked how accurately the model can restore canonical SMILES for these molecules. We intentionally selected the generated SMILES keeping in mind possible applications of the proposed method in artificial intelligence-driven pipelines of de-novo drug development. The model correctly canonicalized $83.6 \%$ of all samples, Table 2.

\section{QSAR modeling}

For the QSAR modelling the saved embedding was used. The training was done using a fixed learning rate $\lambda=0.001$ for $\mathrm{n}=100$ epochs. Early stopping with $10 \%$ randomly selected SMILES was used to identify the optimal model. Table 3, Fig. 6 compares results for regression datasets

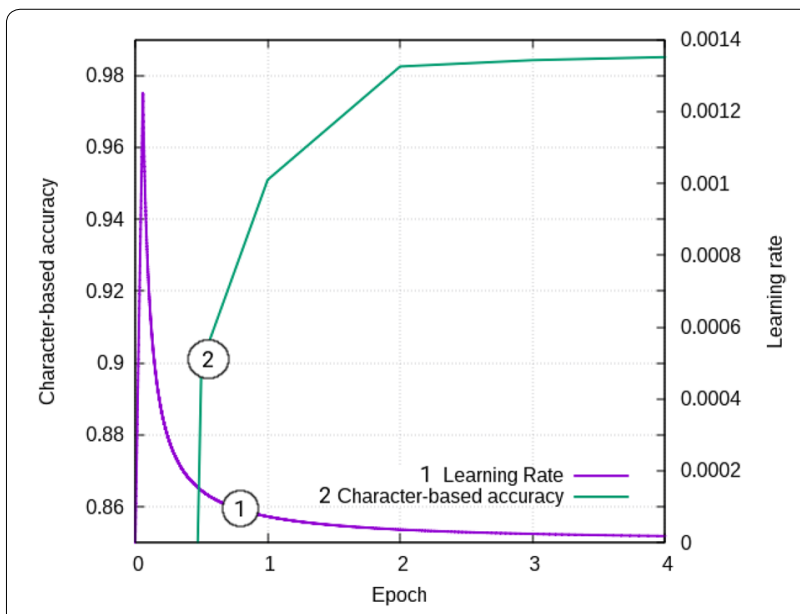

Fig. 5 Learning curves: 1) learning rate schedule (axes bottom and right), and 2) character-based accuracy (axes bottom and left) on the training dataset for the first four epochs
Table 2 Validation of canonicalization model

\begin{tabular}{lrr}
\hline Strings & All & Correctly canonicalized \\
\hline All & 500,000 & $418,233(83.6 \%)$ \\
Stereo (with @) & 77,472 & $28,821(37.2 \%)$ \\
Cis/trans (with / or V) & 54,727 & $40,483(73.9 \%)$ \\
\hline
\end{tabular}

while Table 4, Fig. 7 compares classification tasks. The standard mean errors of the values were calculated using a bootstrap procedure as explained elsewhere [53].

With an exception of a few datasets, the proposed method provided similar or better results than those calculated using descriptor-based approaches as well as the other SMILES-based approaches investigated in our previous study [43]. The data augmentation was critically important for the Transformer-CNN method to achieve its high performance. We used augmentation $n=10$, i.e., 10 SMILES were randomly generated and used for model development and application, which was found optimal in the aforementioned previous study.

Similar to Transformer-CNN the Sml2canSml used an internal representation, which was developed by mapping arbitrary SMILES to canonical SMILES. The difference was that Sml2canSml generated a fixed set of 512 latent variables (CDDD descriptors), while the Transformer$\mathrm{CNN}$ representation had about the same length as the initial SMILES. Sml2canSml CDDD could be used as descriptors for any traditional machine learning methods while Transformer-CNN required convolutional neural networks to process the variable length output and to correlate it with the analysed properties. Sml2canSml was added as CDDD descriptors to OCHEM. These descriptors were analysed by the same methods as used in the previous work, i.e., LibSVM [57], Random Forest [58], XGBoost [59] as well as by Associative Neural Networks (ASNN) [60] and Deep Neural Networks [61]. Exactly the same protocol, fivefold cross-validation, was used for all calculations. The best performance using the CDDD descriptors was obtained by ASNN and LibSVM methods, which contributed models with the highest accuracy for seven and five datasets respectively (LibSVM method provided the best performance in the original study). Transformer-CNN provided better or similar results compared to the CDDD descriptors for all datasets with an exception of Lipo and FreeSolv. It should be also mentioned that CDDD descriptors could only process molecules which satisfy the following conditions:

$\log \mathrm{P} \in(-5,7)$ and

mol_weight $\in(12,600)$ and

num_heavy_atoms $\in(3,50)$ and

molecule is organic. 
Table 3 Coefficient of determination, $\mathbf{r}^{2}$, calculated for regression sets (higher values are better)

\begin{tabular}{llllll}
\hline Dataset & $\begin{array}{l}\text { Descriptor based } \\
\text { methods }^{2}\end{array}$ & $\begin{array}{l}\text { SMILES based } \\
\text { (augm = 10) }\end{array}$ & $\begin{array}{l}\text { Transformer-CNN, } \\
\text { no augm }\end{array}$ & $\begin{array}{l}\text { Transformer-CNN, } \\
\text { augm = 10 }\end{array}$ & $\begin{array}{l}\text { CDDD descriptors } \\
\text { b }\end{array}$ \\
\hline MP & 0.83 & 0.85 & 0.83 & 0.86 & 0.85 \\
BP & 0.98 & 0.98 & 0.97 & 0.98 & 0.98 \\
BCF & 0.85 & 0.85 & $0.71 \pm 0.02$ & 0.85 & 0.81 \\
FreeSolv & 0.94 & 0.93 & $0.72 \pm 0.02$ & 0.91 & 0.93 \\
LogS & 0.92 & 0.92 & 0.85 & 0.91 & 0.91 \\
Lipo & 0.7 & 0.72 & 0.6 & 0.73 & 0.74 \\
BACE & 0.73 & 0.72 & 0.66 & 0.76 & 0.75 \\
DHFR & $0.62 \pm 0.03$ & $0.63 \pm 0.03$ & $0.46 \pm 0.03$ & $0.67 \pm 0.03$ & $0.61 \pm 0.03$ \\
LEL & $0.19 \pm 0.04$ & $0.25 \pm 0.03$ & $0.2 \pm 0.03$ & $0.27 \pm 0.04$ & $0.23 \pm 0.04$ \\
\hline
\end{tabular}

We omitted the standard mean errors, which are 0.01 or less, for the reported values

a Results from our previous study [22]. ${ }^{\mathrm{b}}$ Best performance calculated with CDDD descriptors obtained using autoencoder Sml2canSml from [27]

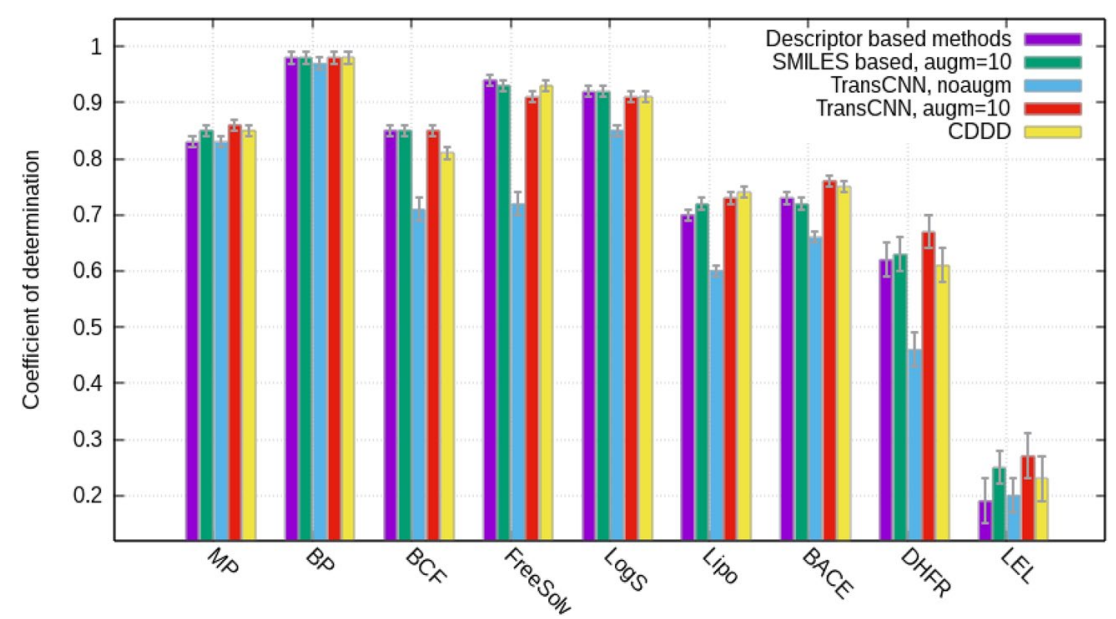

Fig. 6 Coefficient of determination, $r^{2}$, calculated for regression sets (higher values are better)

Table 4 AUC calculated for classification sets (higher values are better)

\begin{tabular}{llllll}
\hline Dataset & $\begin{array}{l}\text { Descriptor based } \\
\text { methods }^{\mathbf{a}}\end{array}$ & $\begin{array}{l}\text { SMILES based } \\
\left(\mathbf{a u g m}=\mathbf{1 0} \mathbf{~}^{\mathbf{2}}\right.\end{array}$ & $\begin{array}{l}\text { Transformer-CNN, } \\
\text { no augm }\end{array}$ & $\begin{array}{l}\text { Transformer-CNN, } \\
\text { augm }=10\end{array}$ & $\begin{array}{c}\text { CDDD descriptors } \\
\text { b }\end{array}$ \\
\hline HIV & 0.82 & 0.78 & 0.81 & 0.83 & 0.74 \\
AMES & 0.86 & 0.88 & 0.86 & 0.89 & 0.86 \\
BACE & 0.88 & 0.89 & 0.89 & 0.91 & 0.9 \\
Clintox & $0.77 \pm 0.03$ & $0.76 \pm 0.03$ & $0.71 \pm 0.02$ & $0.77 \pm 0.02$ & $0.73 \pm 0.02$ \\
Tox21 & 0.79 & 0.83 & 0.81 & 0.82 & 0.82 \\
BBBP & 0.90 & 0.91 & 0.9 & 0.92 & 0.89 \\
JAK3 & $0.79 \pm 0.02$ & $0.8 \pm 0.02$ & $0.70 \pm 0.02$ & $0.78 \pm 0.02$ & $0.76 \pm 0.02$ \\
BioDeg & 0.92 & 0.93 & 0.91 & 0.93 & 0.92 \\
RP AR & 0.85 & 0.87 & 0.83 & 0.87 & 0.86 \\
\hline
\end{tabular}

We omitted the standard mean errors, which are 0.01 or less, for the reported values

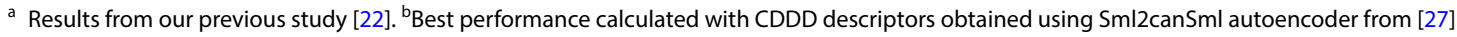




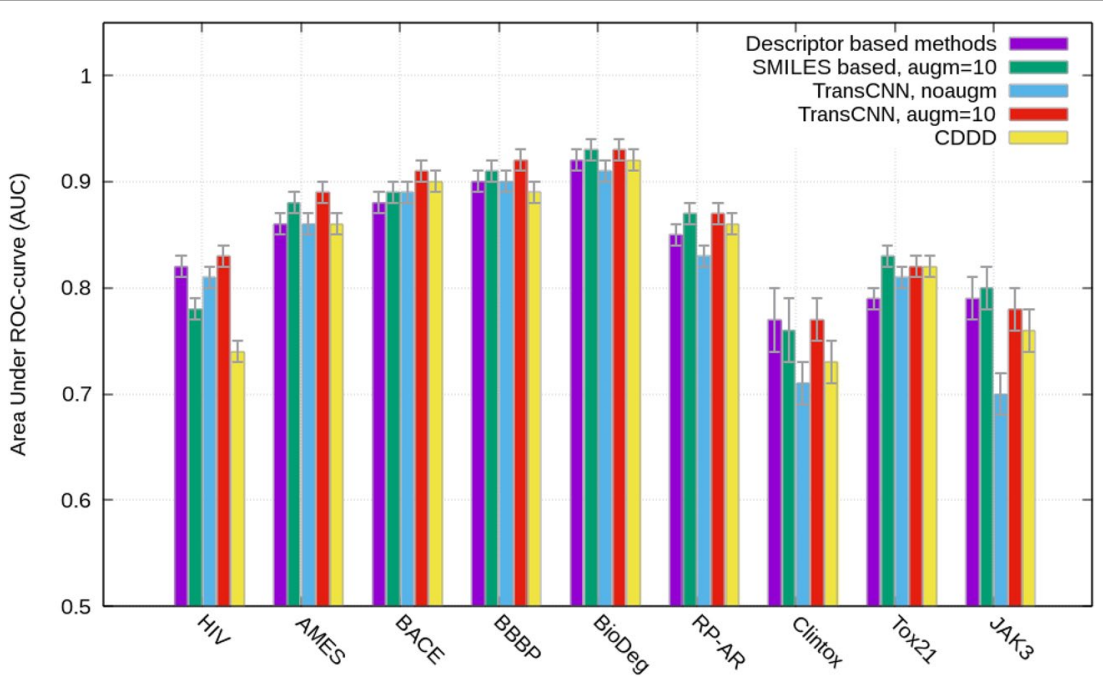

Fig.7 AUC calculated for classification sets (higher values are better)

These limitations appeared due to the preparation of the training set to develop the Sml2canSml encoder. The limitations resulted in the exclusion of a number of molecules, which failed one or several of the above conditions. Contrary to the Sml2canSml encoder, we trained Transformer-CNN with very diverse molecules from ChEMBL and thus the developed models could be applied to any molecule which can be processed by RDKiT. The exclusion of molecules for which CDDD descriptors failed to be calculated did not significantly change the results of Transformer models: some models improved while others decreased their accuracy for $\sim 0.01$ respective performance values. For example, for Lipo and FreeSolv sets the accuracy of the Transformer-CNN model increased to $\mathrm{r}^{2}=0.92$ and 0.75 respectively, while for BBB the AUC decreased to 0.91 .

\section{Interpretability of the model}

Layer-wise relevance propagation was used to interpret the models. For gated connections (in HighWay block) we implemented the signal-take-all redistribution rule [62] while all other Dense and Convolutional layers were well fitted in the LRP framework [32] without any adaptation. In this work, we stopped the relevance propagation on the output of the Transformer's encoder which is positionwise. It should be noted that we froze the encoder part of the network during QSAR model training. Summing up all the individual features for each position in the SMILES string calculated its contribution to the final result. If the LRP indicated a reasonable explanation of the contributions of fragments then one can trust that the model made predictions based on detected fundamental structure-property relationships. For explanation we selected classification (AMES mutagenicity) and regression (water solubility) models.

\section{AMES mutagenicity}

The AMES test is a widely used qualitative test to determine the mutagenic potential of a molecule, from which extensive structural alerts collections were derived [63]. Examples of these alerts are aromatic nitros, N-oxides, aldehydes, monohaloalkenes, quinones, etc. A QSAR model for AMES had to pay special attention to these and similar groups to be interpretable and reliable. The Transformer-CNN model built on 6542 endpoints (3516 mutagenic and 3026 nonmutagenic) results in $\mathrm{AUC}=0.89$, Table 4.

The structure of 1-Bromo-4-nitrobenzene gave the positive AMES test. The output of the LRP procedure for one of possible SMILES for this compound, namely $1 \mathrm{c}([\mathrm{N}+]$ $([\mathrm{O}-])=\mathrm{O}) \mathrm{ccc}(\mathrm{c} 1) \mathrm{Br}$, is shown in Table 5 .

According to the LRP, the relevance was constant during the propagation:

$$
\begin{aligned}
y & =R=f(x)=\sum_{l \in(L)} R_{l}=\sum_{l \in(L-1)} R_{l} \\
& =\sum_{l \in(L-2)} R_{l}=\sum_{l \in(1)} R_{l} .
\end{aligned}
$$

Here (L) stood for a set of neurons in the last layer, $(\mathrm{L}-1)$ - in the layer before the last layer, and so on. Each layer in the Transformer-CNN network contained biases (B), and thus some relevance dissipated on them. Therefore the above equation was corrected to:

$$
\sum_{l \in(L)} R_{l}=\sum_{l \in(L-1)} R_{l}+B
$$


Table 5 Local relevance conservation for $\mathrm{c} 1 \mathrm{c}([\mathrm{N}+]([\mathrm{O}-])=0) \mathrm{ccc}(\mathrm{Br}) \mathrm{c1}$

\begin{tabular}{lcclc}
\hline Layer & Relevance, R (L+1) & Relevance, R (L) & Delta, R (L+ 1)-R (L) & $\begin{array}{l}\text { Bias, Delta / R } \\
(\mathbf{L}+\mathbf{1}) * \mathbf{1 0 0 \%}\end{array}$ \\
\hline Result & & & - & - \\
HighWay Output & 0.98119 & - & 0.0512 & 5.21 \\
HighWay Input & 0.98119 & 0.9300 & 0.2073 & 22.3 \\
DeMaxPool & 0.9300 & 0.7227 & -0.0144 & -1.98 \\
Conv1 & 0.7227 & 0.7371 & -0.00271 & -30.1 \\
Conv2 & 0.0090 & 0.0117 & $0^{\mathrm{a}}$ & 0 \\
Conv3 & 0.1627 & 0.1627 & 0 & 0 \\
Conv4 & -0.0443 & -0.0443 & 0 & 0 \\
Conv5 & 0.0191 & 0.0191 & 0 & 0 \\
Conv6 & -0.0984 & -0.0984 & 0 & 0 \\
Conv7 & -0.0136 & -0.0136 & 0 & 0 \\
Conv8 & 0.0806 & 0.0806 & 0 & 0 \\
Conv9 & 0.0957 & 0.0957 & 0 & 0 \\
Conv10 & 0.1528 & 0.1528 & 0 \\
Conv15 & 0.0845 & 0.0845 & 0 & 0 \\
Conv20 & 0.1038 & 0.1038 & 0 & 24.6 \\
Total & 0.1851 & 0.1851 & 0.2414 & 0 \\
\hline
\end{tabular}

a All 0 values were all less than $10^{-5}$

We calculated how much of the relevance was taken by biases and reported these values in the output of the ochem.py script. Table 5 clearly shows that $24.6 \%$ of the output signal was taken by biases and $75.4 \%$ were successfully propagated to position-wise layers, which we used to interpret the model. If less than $50 \%$ of the signal came to the input, it may indicate an applicability domain problem or technical issues with relevance propagation. In these cases the interpretation could be questioned.

Iterating through all non-hydrogen atoms, the interpretation algorithm picked up an atom and drew a SMILES from it. Thus, every molecule had a corresponding set of SMILES equal to the number of atoms. The LRP was used for every SMILES, and then the individual predictions were averaged for the final output. 1-Bromo-4-nitrobenzene was predicted as mutagenic with the score 0.88 . Impacts of the atoms on the property is depicted in Fig. 8. The model predicted this compound as mutagenic because of the presence of nitro and halogen benzene moieties. Both are known to be structural alerts for mutagenicity [63]. Charged oxygen provided a bigger impact than the double bonded one in the nitro group because its presence contributed to the mutagenicity for nitro and also for N-oxide compounds.

\section{Aqueous solubility}

Solubility is a crucial property in drug-development. To have a fast, robust, and explainable tool for its prediction and interpretation is highly desirable by both academia

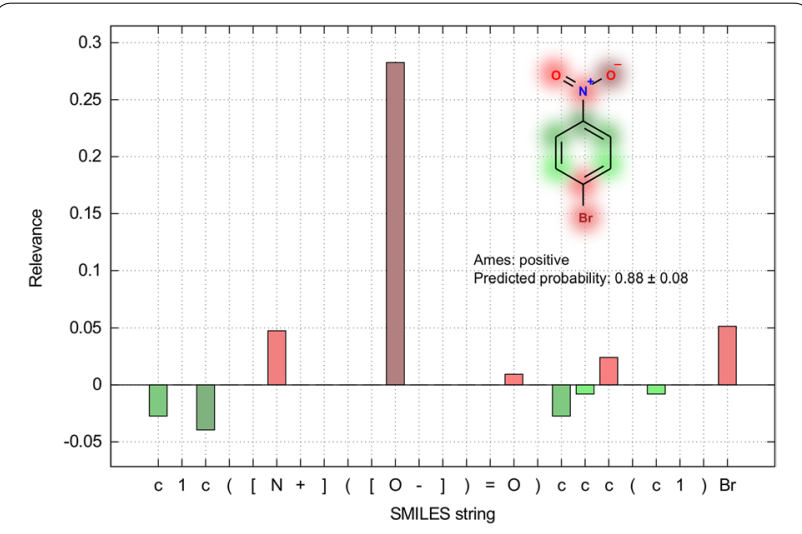

Fig. 8 Visualization of atom contributions, in the case of a mutagenic compound. The red color stands for mutagenic alerts, color green against it

and industry. The Transformer-CNN model built on 1311 compounds had the following statistics: $q^{2}=0.92$ and $R M S E_{p}=0.57$ [64]. For demonstration of its interpretability we choose haloperidol-a well-known antipsychotic drug with $14 \mathrm{mg} / \mathrm{l}$ water solubility [65].

The Transformer model calculated the same solubility $14 \pm 2 \mathrm{mg} / \mathrm{L}$ for this compound. The individual atom contributions are shown in Fig. 9. Hydroxyl, carbonyl, aliphatic nitrogen, and halogens contributed mostly to the solubility. These groups can form ionizable zones in the molecule thus helping water to dissolve the substance. 


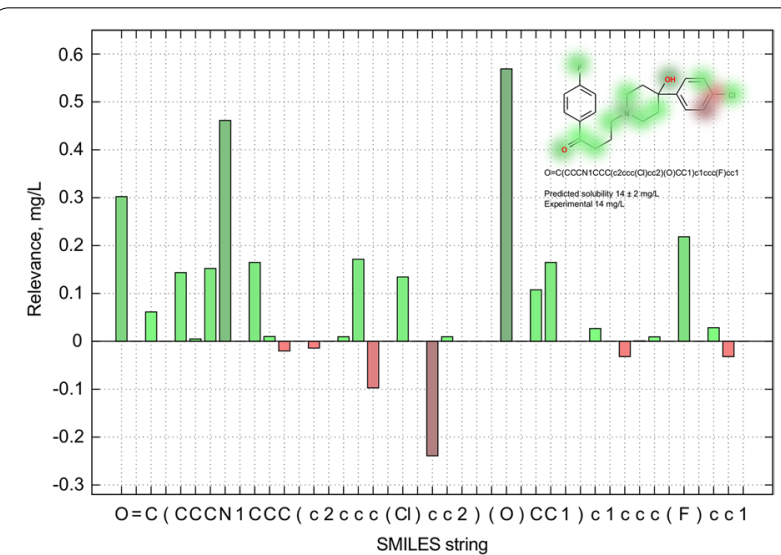

Fig. 9 Visualization of atom contributions to aqueous solubility of haloperidol. The greep bars stand for more soluble features, whereas the red ones show the opposite effect

Several aromatic carbons had negative contributions, which was expected since aromatic compounds are poorly soluble in water. Thus the overall explanation made sense, and the model had an excellent statistics not because of spurious correlations, but because it found the right fragmental features responsible for modelled property. The standalone program contributed in this work has no dependencies on machine learning frameworks, it is easy to install, to use, and to interpret the modelling results. This will make it an indispensable work-horse for drugdevelopment projects world-wide.

\section{Conclusions and outlook}

For the first time we proposed a SMILES canonicalization method based on Transformer architecture that extracts information-rich real-value embeddings during the encoding process and exposes them for further QSAR studies. Also, for the first time we developed a framework for the interpretation of models based on the Transformer architecture using a layer-wise relevance propagation (LPR) approach.

TextCNN approaches efficiently worked with embeddings generated by Transformer, and the final quality of the QSAR models was higher compared to the models obtained with the state-of-the-art methods on the majority of diverse benchmark datasets. The Transformer-CNN architecture required less than a hundred iterations to converge for QSAR tasks to model various biological activity or physico-chemical properties. It can be easily embedded into de-novo drug development pipelines. The model predictions interpreted in a fragment contribution manner using the LPR could be useful to design new molecules with desired biological activity and ADMETox properties. The source code is available on https://githu b.com/bigchem/transformer-cnn as well as an on-line version on https://ochem.eu. For solubility and AMES mutagenicity we also deposited standalone models in the GitHub repository, which not only predict the respective properties but also provide interpretations of predictions.

The Transformer-CNN predicts the endpoint based on an average of individual prognosis for a batch of augmented SMILES belonging to the same molecule. The deviation within the batch can serve as a measure of a confidence interval of the prognosis. Dissipation of relevance on biases as well as analysis of restored SMILES can be used to derive the applicability domains of models. These questions will be addressed in the upcoming studies.

Also, as a comment, we do not think that the authors benchmarking their methods are impassioned about their work. Such benchmarking could be properly done by other users, and we do hope to see the proposed method used soon in future publications. But indeed, remarkably, in this work we saw an outstanding performance of the proposed architecture, which provided systematically better or at least similar results compared to the best descriptor-based approaches as well as several analysed deep neural network architectures. Even more remarkably, the Transformer CNN has practically no adjustable meta parameters and thus does not require spending time to tune hyperparameters of neural architectures, use the grid search to optimise Support Vector Machines, optimise multiple parameters of XGBoost, apply various descriptors filtering and preprocessing, which could easily contribute to the overfitting of models. This as well as the possibility to interpret models makes Transformer $\mathrm{CNN}$ a Swiss-knife for QSAR modeling and interpretation, which will help to make the QSAR great again!

\section{Abbreviations \\ ADMETox: Absorption, distribution, metabolism, excretion and toxicity; ANN: Artificial neural network; CNN: Convolutional neural network; LSTM: Long Short-Term memory; OCHEM: On-line chemical database and modeling envi- ronment; SMILES: Simplified Molecular-Input Line-Entry System; QSAR/QSPR: Quantitative Structure Activity/Property Relationship; RF: Receptive field; RNN Recurrent Neural Network; CNN: Convolutional Neural Network; Transformer- CNN: Transformer Convolutional Neural Network.}

\section{Acknowledgments}

The authors thank NVIDIA Corporation for donating Quadro P6000, Titan Xp, and Titan $\mathrm{V}$ graphics cards for this research work.

\section{Authors' contributions}

PK implemented the method, IVT and GC performed the analysis and benchmarking. All authors read and approved the final manuscript.

\section{Funding}

This study was funded by the European Union's Horizon 2020 research and innovation program under the Marie Skłodowska-Curie grant agreement No. 676434, "Big Data in Chemistry" and ERA-CVD "CardioOncology" project, BMBF 01KL1710. 


\section{Availability of data and materials}

The source code of the Transformer-CNN is available on https://github.com/ bigchem/transformer-cnn. Ready-to-use implementation, training datasets, and models are available on OCHEM https://ochem.eu.

\section{Competing interests}

The authors declare that they have no actual or potential conflicts of interests.

\section{Author details}

${ }^{1}$ Institute of Structural Biology, Helmholtz Zentrum München-Research Center for Environmental Health $(\mathrm{GmbH})$, Ingolstädter Landstraße 1, 85764 Neuherberg, Germany. ${ }^{2}$ BIGCHEM GmbH, Ingolstädter Landstraße 1, 85764 Neuherberg, Germany. ${ }^{3}$ Firmenich International SA, Digital Lab, Geneva, Lausanne, Switzerland.

Received: 12 October 2019 Accepted: 9 March 2020 Published online: 18 March 2020

\section{References}

1. Vaswani A, Shazeer N, Parmar N, et al (2017) Attention is all you need. Paper presented at the 31st Conference on Neural Information Processing Systems (NIPS 2017), Long Beach, CA, USA. arXiv:1706.03762

2. Zhang $X$, Zhao J, LeCun Y (2015) Character-level convolutional networks for text classification. arXiv e-prints. arXiv:1509.01626

3. Sushko I, Novotarskyi S, Körner R et al (2011) Online chemical modeling environment (OCHEM): web platform for data storage, model development and publishing of chemical information. J Comput Aided Mol Des 25:533-554. https://doi.org/10.1007/s10822-011-9440-2

4. Mauri A, Consonni V, Pavan M, Todeschini R (2006) Dragon software: an easy approach to molecular descriptor calculations. Match 56:237-248

5. Baskin I, Varnek A (2008) Fragment descriptors in SAR/QSAR/QSPR studies, molecular similarity analysis and in virtual screening. Chemoinformatics approaches to virtual screening. Royal Society of Chemistry, Cambridge, pp 1-43

6. Eklund M, Norinder U, Boyer S, Carlsson L (2014) Choosing feature selection and learning algorithms in QSAR. J Chem Inf Model 54:837-843. https ://doi.org/10.1021/ci400573c

7. Baskin II, Winkler D, Tetko IV (2016) A renaissance of neural networks in drug discovery. Expert Opin Drug Discov 11:785-795. https://doi. org/10.1080/17460441.2016.1201262

8. Duvenaud D, Maclaurin D, Aguilera-Iparraguirre J, et al (2015) Convolutional networks on graphs for learning molecular fingerprints. arXiv e-prints. arXiv:1509.09292

9. Coley CW, Barzilay R, Green WH et al (2017) Convolutional embedding of attributed molecular graphs for physical property prediction. J Chem Inf Model 57:1757-1772. https://doi.org/10.1021/acs.jcim.6b00601

10. Gómez-Bombarelli R, Wei JN, Duvenaud D et al (2018) Automatic chemical design using a data-driven continuous representation of molecules. ACS Central Sci 4:268-276. https://doi.org/10.1021/acscentsci.7b00572

11. Kimber TB, Engelke S, Tetko IV, et al (2018) Synergy effect between convolutional neural networks and the multiplicity of smiles for improvement of molecular prediction. arXiv e-prints. arXiv:1812.04439

12. Gilmer J, Schoenholz SS, Riley PF, et al (2017) Neural message passing for quantum chemistry. Proceedings of the 34 th International conference on machine learning, Sydney, Australia, PMLR 70. arXiv:1704.01212

13. Shang C, Liu Q, Chen K-S, et al (2018) Edge attention-based multi-relational graph convolutional networks. arXiv e-prints. arXiv:1802.04944

14. Weininger D (1988) SMILES, a chemical language and information system. 1. Introduction to methodology and encoding rules. J Chem Inf Comput Sci 28:31-36. https://doi.org/10.1021/ci00057a005

15. O'Boyle NM, Banck M, James CA et al (2011) Open babel: an open chemical toolbox. J Cheminform 3:33. https://doi.org/10.1186/1758-2946-3-33

16. Vidal D, Thormann M, Pons M (2005) LINGO, an efficient holographic text based method to calculate biophysical properties and intermolecular similarities. J Chem Inf Model 45:386-393. https://doi.org/10.1021/ci049 6797

17. Zhang $X$, LeCun $Y$ (2015) Text understanding from scratch. arXiv e-prints. arXiv:1502.01710
18. Goh GB, Hodas NO, Siegel C, Vishnu A (2017) SMILES2Vec: an interpretable general-purpose deep neural network for predicting chemical properties. arXiv e-prints. arXiv:1712.02034

19. Jastrzębski S, Leśniak D, Czarnecki WM (2016) Learning to SMILE(S). arXiv e-prints. arXiv:1602.06289

20. Goh GB, Siegel C, Vishnu A, Hodas NO (2017) Using rule-based labels for weak supervised learning: a chemnet for transferable chemical property prediction. arXiv e-prints. arXiv:1712.02734

21. Zheng S, Yan X, Yang Y, Xu J (2019) Identifying structure-property relationships through SMILES syntax analysis with self-attention mechanism. J Chem Inf Model 59:914-923. https://doi.org/10.1021/acs.jcim.8b00803

22. Tetko IV, Karpov P, Bruno E, Kimber TB, Godin G. Augmentation Is What You Need! In: Tetko IV, Karpov P, Kurkova V (ed) 28th International Conference on Artificial Neural Networks Munich, Germany, 2019 Sep 17, Proceedings, Part V, Workshop and Special sessions, Springer, Cham, pp 831-835

23. Kiela D, Bottou L (2014) Learning image embeddings using convolutional neural networks for improved multi-modal semantics. In: Proceedings of the 2014 Conference on empirical methods in natural language processing (EMNLP). pp 36-45

24. Pennington J, Socher R, Manning CD (2014) Glove: global vectors for word representation. EMNLP

25. Hinton GE, Salakhutdinov RR (2006) Reducing the dimensionality of data with neural networks. Science 313:504-507. https://doi.org/10.1126/scien ce. 1127647

26. Heller S, McNaught A, Stein S et al (2013) InChl - the worldwide chemical structure identifier standard. J Cheminform 5:7. https://doi. org/10.1186/1758-2946-5-7

27. Winter R, Montanari F, Noé F, Clevert D-A (2019) Learning continuous and data-driven molecular descriptors by translating equivalent chemical representations. Chem Sci 10:1692-1701. https://doi.org/10.1039/c8sc0 $4175 j$

28. Hochreiter S, Schmidhuber J (1997) Long short-term memory. Neural Comput 9:1735-1780. https://doi.org/10.1162/neco.1997.9.8.1735

29. Schwaller P et al (2019) Molecular transformer: A model for uncertaintycalibrated chemical reaction prediction. ACS Cent Sci 5:1572-1583. https ://doi.org/10.1021/acscentsci.9b00576

30. Karpov P, Godin G, Tetko IV. A transformer model for retrosynthesis. In: Tetko IV, Theis F, Karpov P, Kurkova V (ed) 28th International Conference on artificial neural networks, Munich, Germany, September 17-19, 2019 Proceedings, Part V, Workshop and Special sessions. Springer

31. SamekW, Müller K-R (2019) Towards explainable artificial intelligence. In: Samek W, Montavon G, Vedaldi A, et al. (eds) Explainable Al: interpreting explaining and visualizing deep learning. Springer International Publishing, Cham, pp 5-22

32. Montavon G, Binder A, Lapuschkin S et al (2019) Layer-wise relevance propagation: an overview. In: Samek W, Montavon G, Vedaldi A, et al. (eds) Explainable Al: interpreting, explaining and visualizing deep learning. Springer International Publishing, Cham, pp 193-209

33. Tetko IV, Villa AE, Livingstone DJ (1996) Neural network studies. 2. Variable selection. J Chem Inf Comput Sci 36:794-803. https://doi.org/10.1021/ ci950204c

34. Gaulton A, Bellis LJ, Bento AP et al (2012) ChEMBL: a large-scale bioactivity database for drug discovery. Nucleic Acids Res 40:D1100-D1107. https:// doi.org/10.1093/nar/gkr777

35. Segler MHS, Kogej T, Tyrchan C, Waller MP (2017) Generating focussed molecule libraries for drug discovery with recurrent neural networks

36. Gupta A, Múller AT, Huisma BJH et al (2018) Generative recurrent networks for de novo drug design. Mol Inform 37:1700111

37. Rush A (2018) The annotated transformer. In: Proceedings of workshop for NLP open source software (NLP-OSS). pp 52-60

38. Abadi M, Barham P, Chen J, et al (2016) TensorFlow: a system for largescale machine learning

39. Landrum G RDKit: Open-source cheminformatics. https://www.rdkit.org

40. Ramsundar B, Eastman P, Walters P, Pande V (2019) Deep learning for the life sciences: applying deep learning to genomics, microscopy, drug discovery, and more. O'Reilly Media Inc, Sebastopol

41. Srivastava N, Hinton G, Krizhevsky A et al (2014) Dropout: a simple way to prevent neural networks from overfitting. J Mach Learn Res 15:1929-1958

42. Srivastava RK, Greff K, Schmidhuber J (2015) Highway Networks. Paper presented at the Deep Learning Workshop, International Conference on Machine Learning, Lille, France. arXiv:1505.00387 
43. Tetko IV, Karpov P, Bruno E, et al (2019) Augmentation Is What You Need!: 28th International Conference on artificial neural networks, Munich, Germany, September 17-19, 2019, Proceedings. In: Tetko IV, Kůrková V, Karpov $P$, Theis F (eds) Artificial neural networks and machine learning-ICANN 2019: workshop and special sessions. Springer International Publishing Cham, pp 831-835

44. Draper NR, Smith H (2014) Applied regression analysis. Wiley, New York

45. Tetko IV, Sushko Y, Novotarskyi S et al (2014) How accurately can we predict the melting points of drug-like compounds? J Chem Inf Model 54:3320-3329. https://doi.org/10.1021/ci5005288

46. Wu Z, Ramsundar B, Feinberg EN et al (2018) MoleculeNet: a benchmark for molecular machine learning. Chem Sci 9:513-530

47. Brandmaier S, Sahlin U, Tetko IV, Öberg T (2012) PLS-optimal: a stepwise d-optimal design based on latent variables. J Chem Inf Model 52:975-983

48. Sushko I, Novotarskyi S, Körner R et al (2010) Applicability domains for classification problems: benchmarking of distance to models for ames mutagenicity set. J Chem Inf Model 50:2094-2111

49. Tetko IV, Tanchuk VY, Kasheva TN, Villa AEP (2001) Estimation of aqueous solubility of chemical compounds using e-state indices. J Chem Inf Comput Sci 41:1488-1493

50. Huuskonen JJ, Livingstone DJ, Tetko IV IV (2000) Neural network modeling for estimation of partition coefficient based on atom-type electrotopological state indices. J Chem Inf Comput Sci 40:947-955

51. Suzuki K, Nakajima H, Saito Y et al (2000) Janus kinase 3 (Jak3) is essential for common cytokine receptor $\gamma$ chain $(\gamma c)$-dependent signaling: comparative analysis of $\gamma c$, Jak3, and $\gamma c$ and Jak3 double-deficient mice. Int Immunol 12:123-132

52. Sutherland JJ, Weaver DF (2004) Three-dimensional quantitative structureactivity and structure-selectivity relationships of dihydrofolate reductase inhibitors. J Comput Aided Mol Des 18:309-331

53. Vorberg S, Tetko IV (2014) Modeling the biodegradability of chemical compounds using the online chemical modeling environment (OCHEM). Mol Inform 33:73-85. https://doi.org/10.1002/minf.201300030

54. Novotarskyi S, Abdelaziz A, Sushko Y et al (2016) ToxCast EPA in vitro to in vivo challenge: insight into the rank-I model. Chem Res Toxicol 29:768-775. https://doi.org/10.1021/acs.chemrestox.5b00481
55. Rybacka A, Rudén C, Tetko IV, Andersson PL (2015) Identifying potential endocrine disruptors among industrial chemicals and their metabolites development and evaluation of in silico tools. Chemosphere 139:372-378

56. Xia Z, Karpov P, Popowicz G, Tetko IV (2019) Focused library generator: case of Mdmx inhibitors. J Comp Aided Mol Des 1:1

57. Chang C-C, Lin C-J (2011) LIBSVM: A library for support vector machines. ACM Transact Int Syst Technol 2:27. https://doi.org/10.1145/19611 89.1961199

58. Breiman L (2001) Random forests. Mach Learn 45:5-32. https://doi. org/10.1023/A:1010933404324

59. Chen T, Guestrin C (2016) XGBoost: A scalable tree boosting system. arXiv [CS.LG]

60. Tetko IV (2002) Associative neural network. Neural Process Lett 16:187199. https://doi.org/10.1023/A:1019903710291

61. Sosnin S, Karlov D, Tetko IV, Fedorov MV (2019) Comparative study of multitask toxicity modeling on a broad chemical space. J Chem Inf Model 59:1062-1072. https://doi.org/10.1021/acs.jcim.8b00685

62. Arras L, Montavon G, Müller K-R, Samek W (2017) Explaining recurrent neural network predictions in sentiment analysis. Proceedings of the 8th workshop on computational approaches to subjectivity, sentiment and social media analysis

63. Plošnik A, Vračko M, Dolenc MS (2016) Mutagenic and carcinogenic structural alerts and their mechanisms of action. Arh Hig Rada Toksikol 67:169-182. https://doi.org/10.1515/aiht-2016-67-2801

64. Xia Z, Karpov P, Popowicz G, Tetko IV (2019) Focused library generator: case of Mdmx inhibitors. J Comput Aided Mol Des. https://doi.org/10.1007/ s10822-019-00242-8

65. Huuskonen J (2000) Estimation of aqueous solubility for a diverse set of organic compounds based on molecular topology. J Chem Inf Comput Sci 40:773-777. https://doi.org/10.1021/ci9901338

\section{Publisher's Note}

Springer Nature remains neutral with regard to jurisdictional claims in published maps and institutional affiliations.
Ready to submit your research? Choose BMC and benefit from:

- fast, convenient online submission

- thorough peer review by experienced researchers in your field

- rapid publication on acceptance

- support for research data, including large and complex data types

- gold Open Access which fosters wider collaboration and increased citations

- maximum visibility for your research: over $100 \mathrm{M}$ website views per year

At $\mathrm{BMC}$, research is always in progress.

Learn more biomedcentral.com/submissions 